\title{
BAW Resonators Based on AlN with Ir Electrodes for Digital Wireless Transmissions
}

\author{
E. Iborra, M. Clement, J. Olivares, S. González- \\ Castilla, and J.Sangrador, \\ Grupo de Microsistemas y Materiales Electrónicos, \\ Universidad Politécnica de Madrid \\ Madrid, Spain \\ eiborra@etsit.upm.es
}

\author{
N. Rimmer and A. Rastogi \\ Aviza Tecnology Inc. \\ Newport, South Wales, UK \\ B. Ivira and A. Reinhardt \\ CEA, Leti, Minatec \\ Grenoble, France
}

\begin{abstract}
We investigate the performance of aluminum nitride (AIN)-based solidly mounted resonators (SMR) made with iridium (Ir) electrodes for applications in WCDMA filters. Ir/AIN/Ir stacks are grown on top of insulating Bragg mirrors composed of alternate $\lambda / 4$ layers of silicon oxycarbide (SiOC) and silicon nitride $\left(\mathrm{Si}_{3} \mathrm{~N}_{4}\right)$. We have developed the technological processes for the fabrication of filters including the trimming of the Ir top electrode for the tuning of the bandwidth. The influence of the thickness of the top electrode after the tuning process in the performance of the SMRs and filters is analyzed. The performance of the devices is compared with that of SMRs and filters of identical design made with Mo electrodes.
\end{abstract}

Keywords-Iridium, AlN, BAW resonator, BAW filter

\section{INTRODUCTION}

Bulk acoustic wave (BAW) resonators based on aluminum nitride (AlN) and derived products, such as bandpass filters and duplexers, are currently being used in consumer products for mobile communication systems (DCS and WCDMA) [1-4]. Two resonator configurations are typically used to enhance the mechanical isolation of the acoustic wave: the suspended film bulk acoustic resonator (FBAR) [5] and the solidly mounted resonator (SMR) [6], the latter being the more suitable for device integration and high RF power handling [7].

Much effort is devoted to improving the performance of SMRs through the investigation of new materials and device configurations allowing increased effective piezoelectric coupling factor $k_{\text {eff }}{ }^{2}$, reduced piezoelectric losses and increased power handling capacity of the devices, in particular to fulfill the large bandwidth requirement of DCS filters. The choice of the metallic electrodes is an especially sensitive task, as they take an active part in many aspects of the SMR performance. Metallic electrodes promoting the growth of highly c-axis oriented AlN films with single polar orientation and contributing to the confinement of the mechanical energy in the piezoelectric layer are the candidates of choice $[8,9]$. Metals most frequently used in BAW applications are $\mathrm{W}$ [10], Pt [6], and $\mathrm{Mo}$ [11], although many others $(\mathrm{Al}, \mathrm{Ta}, \mathrm{Ti}, \mathrm{Cu}, \mathrm{Cr}, \mathrm{Au}, \mathrm{Ru}$, Ir) have been investigated $[9,12,13,14]$. So far, the besttextured AlN films deposited by sputtering have been obtained on Pt and Ir metallic surfaces, in which the $\{111\}$ planes exhibit hexagonal symmetry, providing AIN films with very low values of the full width at half maximum (FWHM) of the rocking-curve (RC) around the $00 \cdot 2$ peak (around $0.9^{\circ}$ for 1 $\mu \mathrm{m}$-thick films) $[8,14]$ and very high piezoelectric activity. Compared to $\mathrm{Pt}$, Ir is considerably cheaper, possesses higher acoustic impedance, and exhibits a lower diffusivity in silicon [15], which is essential in view of its integration with CMOS technologies. Furthermore, our preliminary studies of Ir-based SMRs predicted a good performance in operative filters [16].

In this study we have analyzed the performance of Ir-based SMRs in order to investigate their viability for the fabrication of filters for the $2 \mathrm{GHz}$ range, addressing both the WCDMA and DCS standards. Ir/AIN/Ir stacks of different thickness were used to build resonators and filters of different geometries on top of insulating Bragg mirrors. We present the specific technology developed for the fabrication of Ir-based devices and the preliminary frequency characterization of resonators and filters.

\section{DeVICE FABRICATION}

SMRs were fabricated by growing Ir/AIN/Ir stacks on top of all insulating Bragg mirrors formed by alternating low and high acoustic impedance layers of $\operatorname{SiOC}\left(Z_{\mathrm{a}}=3.6 \times 10^{6} \mathrm{~N} \cdot \mathrm{s} \cdot \mathrm{m}^{-3}\right)$ and $\mathrm{Si}_{3} \mathrm{~N}_{4}\left(Z_{\mathrm{a}}=25.7 \times 10^{6} \mathrm{~N} \cdot \mathrm{s} \cdot \mathrm{m}^{-3}\right)$. Seed layers of $\mathrm{Mo} / \mathrm{Ti}(40: 20$ nm-thick) were used to improve the adhesion and crystal quality of the Ir bottom electrode to the acoustic reflector. Ir slugs (99.98\% pure) were electron-beam evaporated at a base pressure of less than $2 \times 10^{-6}$ Torr to form bottom electrodes of different thicknesses between $80 \mathrm{~nm}$ and $130 \mathrm{~nm}$. The bottom electrode was patterned through a Mo mask by reactive ion etching (RIE), first in pure $\mathrm{Ar}^{+}$to remove the Ir layer and then in $\mathrm{SF}_{6}$ to remove the Mo/Ti seed layer. AlN piezoelectric films were then reactively sputtered with a pulsed DC source in a Sigma fxP cluster tool from Aviza Technology. Before AlN deposition, a $15 \mathrm{~s}$ soft-etch in an Ar discharge was performed to condition the Ir bottom electrode. After degassing the wafers close to the AlN deposition temperature, AlN films were sputtered with a 1:5 Ar/ $\mathrm{N}_{2}$ admixture at a total pressure of 5 mTorr, a pulsed DC power level of $10 \mathrm{~kW}$, and a substrate 
temperature of $400^{\circ} \mathrm{C}$. An RF bias was applied to the substrates to tune the stress in the AlN films to $0 \pm 50 \mathrm{MPa}$. These conditions provided deposition rates of $100 \mathrm{~nm} / \mathrm{min}$ with in-wafer thickness homogeneity of $0.3 \% 1 \sigma$. Ir top electrodes were then deposited and patterned following the same procedure as that used for the bottom electrode. The AIN film was wet-etched in a $\mathrm{KOH}$ solution through a Mo mask to reach the iridium bottom electrode. Finally the pads were re-grown with a $1 \mu \mathrm{m}$ thick Al layer.

Different Ir-based SMRs and filters in the WCDMA band were fabricated following this technology. Some typical configurations are shown in figure 1. The tuning of the filters was achieved by de-loading the low and high frequency resonators by trimming the Ir top electrode. For comparison, Mo-based SMRs and filters were also fabricated with AIN films of identical thickness; in this case the thicknesses of the Mo electrodes were adjusted to achieve the target frequency.

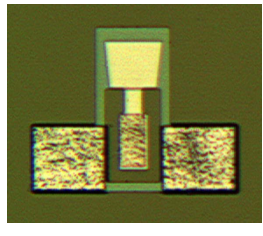

(a)

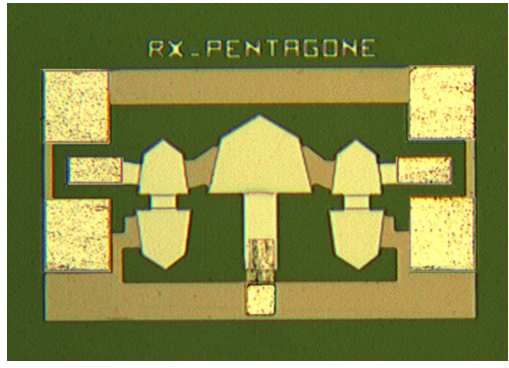

(b)
Figure 1. Optical microscope pictures of (a) typical SMR and (b) typical filter for the WCDMA Rx band.

\section{RESULTS AND DISCUSSION}

\section{A. AlN films assesment}

The crystal structure of the Ir and AlN films was assessed by X-ray diffraction (XRD) by measuring the $\theta / 2 \theta$ patterns and the rocking-curves (RC) around the most intense reflections, i.e. the $\operatorname{AlN} 00 \cdot 2$ at $18.02^{\circ}(\theta)$ and the $\operatorname{Ir} 111$ at $20.35^{\circ}(\theta)$. The surface of the different layers was examined by atomic force microscopy (AFM), which provided the measurement of the surface roughness.

XRD and AFM characterization showed that highly c-axisoriented AlN films were successfully grown on (111)-oriented Ir layers, provided these surfaces were etched with $\mathrm{Ar}+$ ions immediately before AIN deposition (see Fig.2). The pretreatment of the Ir surface was essential to avoid the growth of tilted microcrystals related to the degradation of the piezoelectric response; AIN films with pure $00 \cdot 2$ orientation and $\mathrm{RC}$ ranging from $0.9^{\circ}$ to $3^{\circ}$, almost independent of the texture and roughness of the Ir bottom electrode, were obtained [19]. No degradation of the piezoelectric quality of AlN films with the texture was observed in the mentioned range.

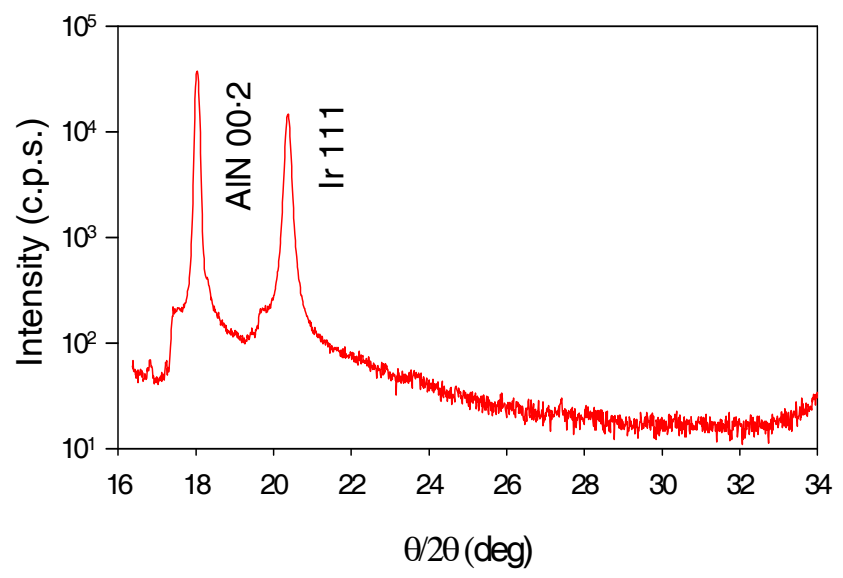

(a)

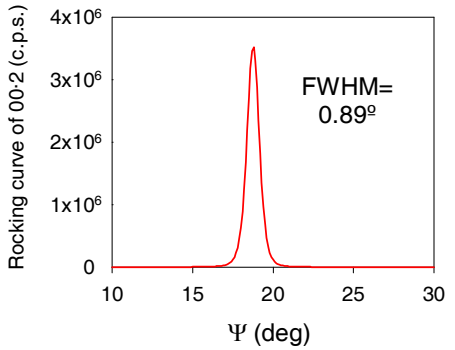

(b)

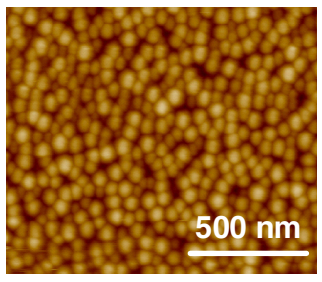

(c)
Figure 2. (a) XRD pattern of a typical AlN film grown on Ir, b) FWHM of the RC around AlN 00.2 peak, and c) AFM image of AlN film surface.

\section{B. Assessment of resonators and filters}

The resonators and filters were characterized by measuring the scattering parameters $\left(S_{\mathrm{ij}}\right)$ at frequencies ranging from $1 \mathrm{GHz}$ to $3 \mathrm{GHz}$, using an Agilent N5230A network analyzer connected to the samples through air-coplanar RF probes from Picoprobe (CGB Industries).

The response of the test resonators was assessed by measuring the electrical reflection coefficient $S_{11}$ from which the complex impedance and admittance were derived. The effective coupling factor of the SMRs $k_{\text {eff }}^{2}$ was determined, according to the standard definition of IEEE [1], from the experimental values of the resonant $\left(f_{\mathrm{r}}\right)$ and antiresonant $\left(f_{\mathrm{a}}\right)$ frequencies of the complex impedance modulus (see figure 3 ), through the expression

$$
k_{e f f}^{2}=\frac{\pi}{2} \frac{f_{r}}{f_{a}} \frac{1}{\tan \left(\frac{\pi}{2} \frac{f_{r}}{f_{a}}\right)}
$$

In the same way, the resonant and antiresonant quality factors $\left(Q_{\mathrm{r}}\right.$ and $\left.Q_{\mathrm{a}}\right)$ were derived experimentally from the slope of the complex impedance phase at the resonant and antiresonant frequencies (see figure 3), respectively [1], through the expression 


$$
Q_{r, a}=\frac{f_{r, a}}{2}\left(\frac{d \angle Z}{d f}\right)_{f_{r, a}}
$$

Fig.3 shows the frequency variation of the modulus and the phase of the complex impedance of a typical Ir-based resonator. It is noticeable the absence of spurii in the response of the resonators indicating a very good acoustic isolation of them from the substrate trough the Bragg mirror.

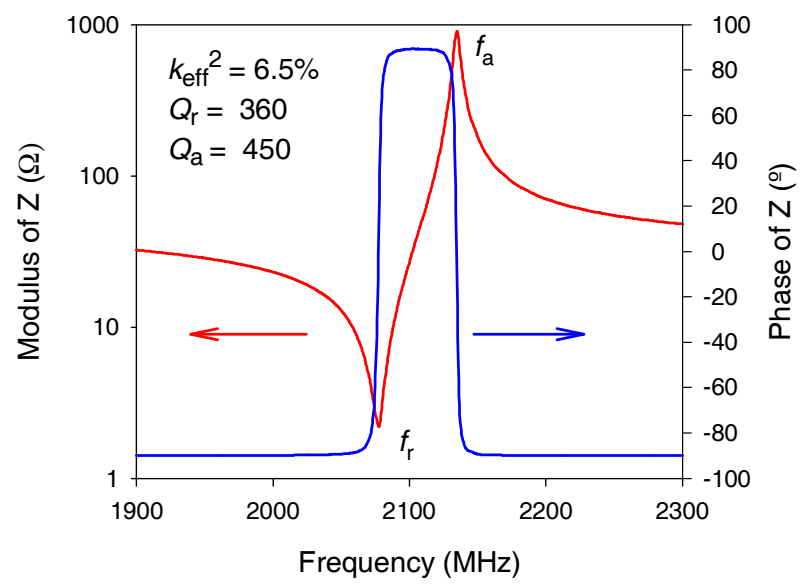

Figure 3. Complex impedance (modulus and phase) of a typical Ir-based resonator as a function of frequency.

The values of $k_{\text {eff }}^{2}, Q_{\mathrm{r}}$ and $Q_{\mathrm{a}}$ were $6.5 \%, 360$ and 450 , respectively. Ir-based resonators exhibited $k_{\mathrm{eff}}{ }^{2}$ values systematically higher than Mo-based resonators of similar geometry and resonant frequency $\left(k_{\text {eff }}^{2}\right.$ around $\left.5.8 \%\right)$. We attribute this fact to the high acoustic impedance of the Ir electrodes, which guarantees a high acoustic impedance mismatch with the AlN film. However, the resonant quality factors $Q_{\mathrm{r}}$ appear to be lower (a typical Mo-based resonator has $Q_{\text {r }}$ up to 800). This is a consequence of the high series resistance of Ir-based resonators, which require metallic electrodes considerably thinner than Mo-based resonators to achieve the same resonant frequency. The series resistance, even after the re-growth of the electrode pads, is higher than that of Mo-based resonators and reduces significantly the $Q_{\mathrm{r}}$ value. An increase of $Q_{\mathrm{r}}$ would require increasing the electrode thicknesses, and thus reducing the AlN thickness accordingly as well as redesigning the filter geometry.

\section{Effects of loading and trimming}

The thickness of the AlN piezoelectric layer was adjusted to achieve a surface capacitance of around $65 \mathrm{pF} / \mathrm{mm}^{2}$. This value was used to design the actual geometry of each resonator inside the filter. The thickness of the bottom electrode was constant whereas the top electrode thickness was adjusted to tune the resonant frequency (in the $1900 \mathrm{MHz}$ to $2200 \mathrm{MHz}$ range for WCDMA Tx and Rx bands).

The bandwidth of ladder filters is achieved by using two kinds of SMRs tuned at different resonant frequencies: the loaded SMR (or shunt SMR), which is the low frequency resonator, and the non-loaded SMR (or series SMR), which constitutes the high frequency resonator. Filters are commonly obtained by adjusting the thickness of the top electrode to achieve the high resonant frequency and then loading the shunt resonators with an additional film (usually $\mathrm{SiO}_{2}$ [8] to adjust the low resonant frequency. The loading of the resonator reduces its $k_{\text {eff }}^{2}$. Theoretical simulations using Mason's model predict that loading the SMRs with heavy materials prevents to some extent the reduction of $k_{\text {eff }}^{2}$. This result was experimentally verified in Ir-based SMRs.

WCDMA filters were fabricated using the following technology. First, the Ir top electrode was grown to a greater thickness than the required to achieve the targeted low resonant frequency. After patterning the top electrode, opening the vias through the AlN film and re-growing the Ir pads with Al, a first trimming, achieved by etching of the top electrode by $\mathrm{Ar}^{+}$ bombardment, was carried out in all resonators to adjust the low resonant frequency. A second trimming of the top electrode of the series SMRs was then performed, while keeping the shunt SMRs masked with a photoresist layer, to fit the high resonant frequency. Individual test SMRs were used to control and characterize the two trimming processes. The advantage of these two "unloading" processes is the use of a single Ir deposition process for all the SMRs. Additionally, etching of Ir with $\mathrm{Ar}^{+}$ions is a well controlled process with a linear frequency shift with etch time, as shown in Fig. 4.

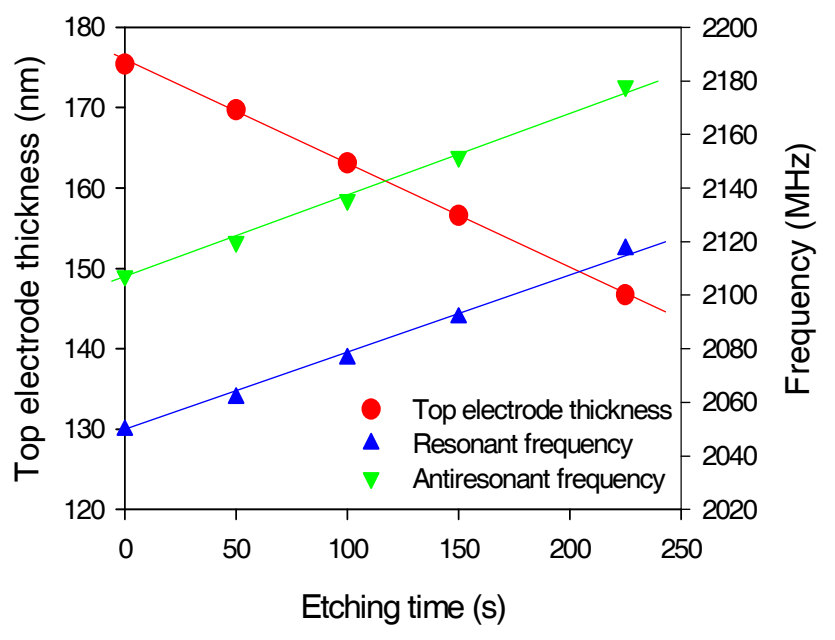

Figure 4. Ir top electrode thickness and resonant frequency vs. etching time

After the trimming processes we verified that the values of $k_{\text {eff }}^{2}, Q_{\mathrm{r}}$ and $Q_{\mathrm{a}}$ measured in the test resonators were barely affected by the thickness reduction of the top electrode. Figure 5 shows the impedance modulus of a typical test SMR for different etching times. The value of $k_{\text {eff }}^{2}$ deduced from the experimental data varied only $2.3 \%$, despite the significant frequency shift of around $270 \mathrm{MHz}$. Similar experiments performed in SMRs loaded with Mo films gave variations of $k_{\text {eff }}^{2}$ of around 5\%, which confirms the advantage of loading the SMRs with heavy materials, such as Ir. 


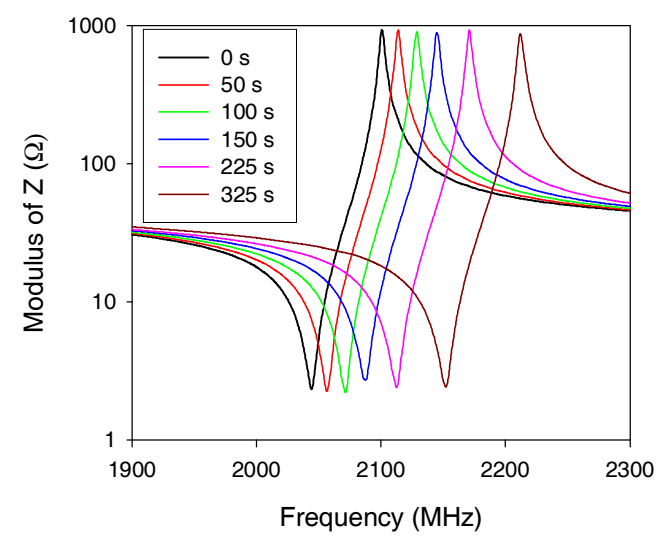

Figure 5. Impedance modulus of a typical Ir-based SMR for different etching times of the top electrode

\section{Frequency response of filters}

Figure 6 shows the frequency response of the preliminary WCDMA Rx filters obtained after trimming the Ir top electrode of the series SMR for different times. It is important to note that that the design of these filters (in terms of geometry, thickness of the different layers, series resistance and impedance matching) was not fully optimized. However, the preliminary results on the behavior of these devices show that filters with high bandwidths can be fabricated with Ir-based SMRs.

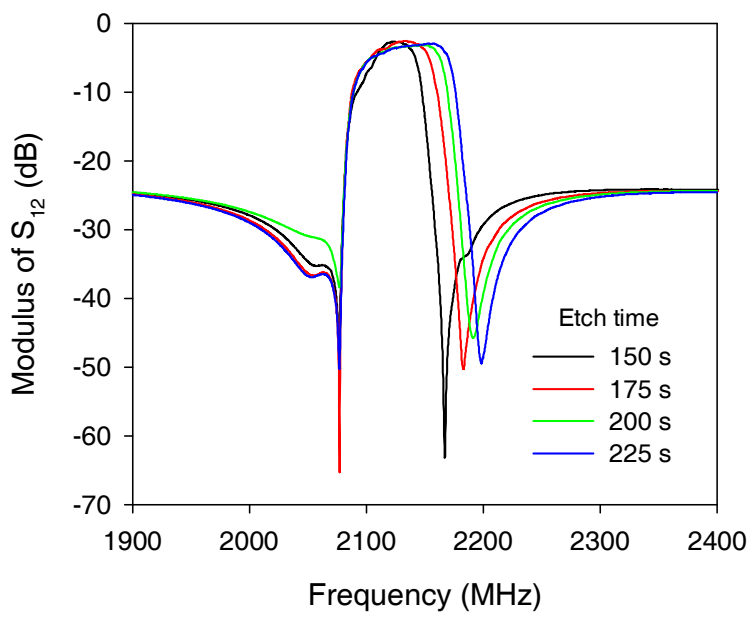

Figure 6. Frequency response of WCDMA Tx filters of different bandwidths obtained afetr different trimmings of the top electrode

\section{CONCLUSIONS}

We have proposed a new material, Ir, to be used as a metallic electrode for AlN-based SMRs and filters. Our choice was based on the very high acoustic impedance of Ir films and their demonstrated ability to promote the growth of AlN films of very high piezoelectric activity. We have also developed the processes for Ir deposition, through electron beam evaporation, and patterning by $\mathrm{Ar}^{+}$bombardment. SMRs, composed of
Ir/AlN/Ir stacks deposited on $\mathrm{SiOC} / \mathrm{Si}_{3} \mathrm{~N}_{4}$ Bragg mirrors, and filters for the $2 \mathrm{GHz}$ range have been fabricated and characterized. The tuning of the resonant frequency of the SMRs, and hence the bandwidth of the filters, was achieved by a simple trimming process of the Ir top electrodes.

The frequency characterization revealed that Ir-based resonators exhibited higher values of $k_{\text {eff }}{ }^{2}$ (up to $7 \%$ in the best devices) than conventional Mo-based resonators, due to the very high acoustic impedance mismatch of Ir and AlN films. Additionally, the values of $k_{\text {eff }}^{2}$ hardly varied with the thickness of the Ir top electrodes, thanks to the very high density of Ir. These two facts along with the simplicity of the Ir electrode trimming offers the possibility of improving the performance of WCDMA filters by increasing their bandwidth without degrading their other characteristics. All this encourages further optimizing the design of Ir-based filters in order to achieve better performing devices.

\section{ACKNOWLEDGEMENT}

This work was partially supported by the European Commission through the 6th Framework Program by the MOBILIS project. (http://www.ist-mobilis.org)

\section{REFERENCES}

[1] R. Aigner, Proc 3rd Int Symposium on Acoustic Wave Devices for Future Mobile Communication Systems, Chiba, Japan, Mar.(2007).

[2] E. Schmidhammer, H. Heinze, M. Schmiedgen, M. Mayer, and A. Link, 2006 IEEE Ultrason. Symp. Proc., 329 (2006).

[3] Jae Y. Park, Hee C. Lee, Kyeong H. Lee, Young J. Ko, and Jong U. Bu, 33rd European Microwave Conference Proc., 907(2003).

[4] A. Volatier, E. Defaÿ, A. N'hari, J. F. Carpentier, P. Ancey, B. Dubus, 2006 IEEE Ultrason. Symp. Proc., 829(2006).

[5] K.M. Lakin, IEEE FCS-EFTF Proc., 1 (2003).

[6] H.P. Loebl, M. Klee, C. Metzmacher, W. Brand, R. Milsomb, and P. Lokc, Mat. Chem. and Phys. 79, 143 (2003).

[7] R. Aigner, N-H. Huynh, M. Handtmann, and S. Marksteiner, IEEE Ultrason. Symp. Proc., 429 (2005).

[8] R. Lanz and P. Muralt, IEEE Trans. Ultrason. Ferr. Freq. Control, 52 (6), 936 (2005).

[9] T. Yokoyama, T. Nishihara, S. Taniguchi, M. Iwaki, Y Satoh, M. Ueda, and T. Miyashita, IEEE Ultrason. Ferr. Freq. Control Proc. 429 (2004).

[10] E. Schimdhammer, B. Bader, W. Sauer, M. Schmiedgen, H. Heinze, C Eggs, and T. Metzger, Microw. Symp. Digest IEEE MTT-S Proc., 233 (2005).

[11] Y-R. Kang, S-C. Kang, K-K. Paek, Y-K. Kim, S-W. Kim and B-K. Ju, Sens. Actuators A 117, 62 (2005).

[12] R. Jakkaraju, G. Henn, C. Shearer, M. Harris, N. Rimmer, and P. Rich, Microelec. Eng. 70, 566 (2003).

[13] M. Akiyama, K. Nagao, N. Ueno, H. Tateyama, T.Yamada, Vacuum 74, 699 (2004).

[14] J. Olivares, M. Clement, E. Iborra, S. González-Castilla, N. Rimmer and A. Rastogi, IEEE Ultrason. Symp. Proc., 1401 (2007).

[15] V. Benda, M. Cernik and D. Stepkova, Microel. J., 29, 695 (1998).

[16] E. Iborra, M. Clement, J. Olivares, J. Sangrador, N. Rimmer and A. Rastogi, IEEE Ultrason. Symp. Proc., 616 (2007). 